\title{
COMPARISON OF SEVERAL REMOTE SENSING IMAGE CLASSIFICATION METHODS BASED ON ENVI
}

\author{
Xingchen $\mathrm{Li}^{1,2}$, Lilong $\mathrm{Liu}^{1,2 *}$, Liangke Huang ${ }^{1,2,3}$
}

1. College of Geomatic Engineering and Geoinformatics, Guilin University, Guilin,541004, China

2. Guangxi Key Laboratory of Spatial Information and Geomatics, Guilin, 541004, China

3. GNSS Research Center, WuHan University, WuHan, 430079, China

KEY WORDS : Supervised Classification,Unsupervised Classification , Accuracy Evaluation, Heze City

\begin{abstract}
:
With the development of remote sensing technology and the increasing accuracy of remote sensing images, research on the accuracy of remote sensing classification is becoming more and more important. However, the classification accuracy obtained by different classification algorithms is also different. To this end, this paper selects the maximum likelihood method and the minimum distance method in the traditional supervised classification, the ISODATA method and the k-means algorithm in the unsupervised classification, and uses these four algorithms to classify the Landsat images in the research area of Heze City. The classification results are obtained and the results are evaluated. Then the four algorithms are compared separately, and the advantages and disadvantages of each algorithm are analyzed. The results show that the classification accuracy of the maximum likelihood method in the supervised classification is relatively high, and the classification accuracy is $82.3281 \%$. The ISODATA algorithm in the supervised classification is superior to the K-means algorithm in clustering effect.
\end{abstract}

*Corresponding author. Address: Guilin University of Technology, Guangxi, China

Email address: hn_liulilong@163.com 


\section{INTRODUCTION}

Remote sensing technology is widely used in the investigation and monitoring of resource environment. The important way to realize this application is remote sensing classification (Yang, Zhou, 2001). Therefore, it is necessary to compare and study remote sensing image classification methods. The literature (Zhang, Huang, 2019) did the research of traditional supervised and unsupervised classification, but did not show the principle of the method in the unsupervised classification; the literature (Tong, 2019) studied the advantages and disadvantages of the supervised classification method, but There is no comparison with unsupervised algorithms; the literature (Wang , Zhang,2019)and(Zhao et al., 2019) uses unsupervised classification to extract winter wheat experiments, and the results are also very good, indicating that unsupervised classification can also meet people's needs and is worthy of study. This paper starts from two kinds of supervised classification and two unsupervised classification algorithms, and studies them according to their principles and compares their advantages and disadvantages.

Remote sensing image classification methods commonly used in remote sensing technology mainly include supervised classification and unsupervised classification (Zhang, 2016). Supervised classification methods require a certain prior knowledge, which is a learning and training process. Non-supervised classification methods do not require priori. Knowledge is a clustering process. These two methods have their own advantages and disadvantages in practical applications.

In this paper, the remote sensing data of Landsat-8 OLI Heze area is used. Through the supervised and unsupervised classification of remote sensing images, the differences between different algorithms can be representatively described and the classification results can be compared. Provides guidance on the selection of classification methods for work related to land classification.

\section{RESEARCH AREA AND DATA}

\subsection{Research area overview}

The research area is selected as Heze City, Shandong Province. Heze City is located in the southwest of Shandong Province. It is 157 kilometers long from north to south, 140 kilometers wide from east to west, and has a total area of 12238.62 square kilometers. It is the junction of the four provinces of Shandong, Jiangsu and Anhui.See Figure 1.The landforms of Heze include river beach highland, gentle slope land, ruin fan-shaped land, ridge highland and dish-shaped depression, among which the slope area is the largest.

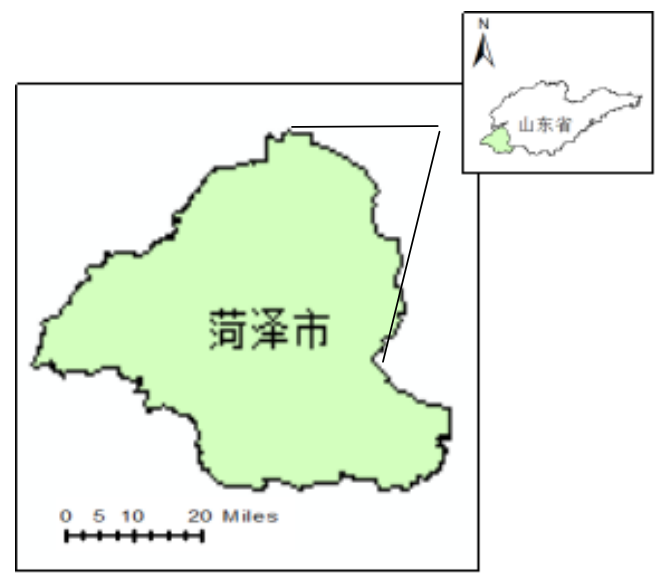

Figure 1. Location of the study area

\subsection{Data sources and pre-processing}

This article uses Landsat-8 OLI image data, the satellite has a total of 11 bands, the band 1-7, 9-11 spatial resolution is 30 meters, the band 8 is 15 meters resolution full color band, OLI land imager There are 9 bands and the imaging width is (185*185) km2. According to the geographical location of the Heze area, it takes 4 scenes to cover the entire area. See Table 1 for details.

\begin{tabular}{|l|c|l|}
\hline $\begin{array}{l}\text { Strip } \\
\text { number/row } \\
\text { number }\end{array}$ & Get time & $\begin{array}{l}\text { Cloud } \\
\text { amount }\end{array}$ \\
\hline $122 / 35$ & $2018-05-03$ T02:47:25 & 0.1 \\
$122 / 36$ & $2018-05-03$ T02:47:48 & 0.13 \\
$123 / 35$ & $2018-05-11$ T02:53:31 & 3.73 \\
$123 / 36$ & $2018-05-11$ T02:53:35 & 3.81 \\
\hline
\end{tabular}

Table 1 Landsat-8OLI image information of Heze area 
Data preprocessing is mainly done by means of ENVI image processing software, including image radiometric calibration, atmospheric correction, geometric fine correction, image mosaic and cropping.

\section{RESEARCH METHODS}

Traditional remote sensing image classification methods are classified into supervised classification and unsupervised classification. Supervised classification is based on prior knowledge to select training samples in the study area. By selecting characteristic parameters, establishing discriminant functions and discriminant rules to classify the images to be classified. Unsupervised classification has no prior knowledge conditions, which is through the remote sensing image itself. Spectral features and statistical features establish decision rules for classification. Both supervised and unsupervised classification methods are applicable to actual work, but their use principles, processes, and range conditions are different.

\subsection{Maximum Likelihood Method}

The maximum likelihood method is based on the probability discriminant function and the Bayesian discriminant rule, which assumes that each type of statistic for each band is normally distributed (Jin, Zhu, 2014). Selecting the region of interest, and obtaining the mean and variance parameters of each category by counting and calculating the region of interest, thereby determining a classification function, and then substituting each pixel in the image to be classified into the classification function of each category, The category of the largest function return value is used as the attribution category of the scanned pixel, so as to achieve the classification effect. Its classification algorithm formula is:

$$
g_{k}\left(x_{i}\right)=\ln p\left(w_{k}\right)-\frac{1}{2} \sum k-\frac{1}{2}\left(x_{i}-m_{i}\right)^{T}\left(\sum k\right)^{-1}\left(x_{i}-\right.
$$
$\left.m_{i}\right)$

In the middle:

$$
\begin{aligned}
& g_{k}\left(x_{i}\right)=\text { Weighted distance } \\
& p\left(w_{k}\right)=\text { Probability that the class to be classified }
\end{aligned}
$$

belongs to category $\mathrm{k}$

$$
\begin{aligned}
& \sum k=\text { Covariance matrix of the kth class } \\
& x_{i}, m_{i}=\text { The measurement vector of the pixel and the }
\end{aligned}
$$

sample mean of the i-th class

\subsection{Minimum distance method}

The original of the minimum distance classification is the point at which each pixel point is assigned to the category center of its nearest distance (Zhao, Qian, 2004). It is based on the Euclidean distance in the feature space as the pixel classification. It requires selecting a representative statistical feature quantity (mean) for each category in the remote sensing image, and first calculating the pixel to be classified and the known category. The Euclidean distance between them, and then classify it as the smallest distance. The distance discriminant function is

$$
\mathrm{D}=\sqrt{\sum_{j=1}^{n}\left(X_{j}-M_{i j}\right)^{2} / S_{i j}^{2}}
$$

In the middle :

$$
\begin{aligned}
& X_{j}=\text { Gray value of pixel } \mathrm{X} \text { in j-band } \\
& \mathrm{n}=\text { Number of bands } \\
& M_{i j}, S_{i j}=\text { Mean and standard deviation of the i-th j-band }
\end{aligned}
$$

\subsection{ISODATA method}

The full name of ISODATA is Iterative Self-organizing Data AnalysisTechnique, which is an iterative self-organizing data analysis technology (Zhao, Zhou, 2016). The ISODATA method is based on the minimum distance center method object meta-clustering. The ISODATA method can select the initial class clustering center according to certain principles, and then calculate the standard deviation of each cluster and the distance between each class center. The smaller the distance, the larger the similarity, the easier it is to belong to the same class. If the cluster standard deviation deviation is greater than the defined threshold, the split is selected. If the class spacing is less than the defined threshold, then merge. The cluster center is continuously calculated and iterated until the average class 
spacing is less than the defined threshold or the average of the two iterative process class spacings is less than the threshold. Its objective function is:

$$
\underset{S}{\operatorname{argmin}} \sum_{i=1}^{k} \sum_{x_{j} \epsilon s_{i}}|| x_{j}-c_{i}||^{2}
$$

In the middle :

$\mathrm{k}=$ The number of categories finally obtained

$\mathrm{S}=$ Classified category collection

$x_{j}$ is each sample belonging to the $S_{i}$ class, $c_{i}$ is the category center of $S_{i}$

\section{4 k-means method}

The K-means clustering algorithm is the most famous partitioning clustering algorithm (Lin, Chen, 2019)and(Qu,et al, 2019), so it is very common to use. The calculation process of the K-means algorithm is very straightforward, that is, randomly extracting $k$ elements from a given set of elements $\mathrm{D}$ as the respective centers of $\mathrm{k}$ clusters, and then calculating the dissimilarity of the remaining elements to the center of the $\mathrm{k}$ clusters, respectively. These elements are assigned to the clusters with the lowest degree of dissimilarity. According to the clustering result, the centers of the k clusters are recalculated. The calculation method is to take the arithmetic mean of the dimensions of all the elements in the cluster, re-cluster all the elements in $\mathrm{D}$ according to the new center, and repeat the re-clustering until the clustering Class results no longer change.Its objective function is the same as equation (3).

\section{CLASSIFICATION PROCESS AND RESULTS EVALUATION}

This study refers to the Chinese land resource classification system established by the Chinese Academy of Sciences and the Ministry of Agriculture, and links the characteristics of Heze City's geographical features and Landsat-8 OLI data to define the training samples of the study area as cultivated land, forest land, construction land, roads, water and other six. Classes, corresponding to the corresponding colors and draw the region of interest.

\subsection{Obtain training samples}

After the image processing of the pre-processed study area is performed by ENVI software, the ROI separability tool is needed to calculate the statistical distance between all categories, and the degree of difference between the two categories is calculated. Separability of the classified samples. The separability is mainly determined by the two parameters of Jeffreys-Matusita distance and Transformed Divergence. See Figure 2. In general, when the parameter value is 1.9-2.0, the sample selection is qualified, and the categories have good separability, greater than 1.8 also meets the requirements can be used. When the parameter value is too small at 0.1-1.0, consider combining the two classes into one class. When it is less than 1.8 , it is necessary to re-select the sample to calculate and obtain the sample that meets the requirements. From the figure, it can be seen that the parameter value between road and construction land, forest land and cultivated land is less than 1.8, because the spectral characteristics between them are very close, so It is necessary to repeat the test and finally obtain the available training samples. 


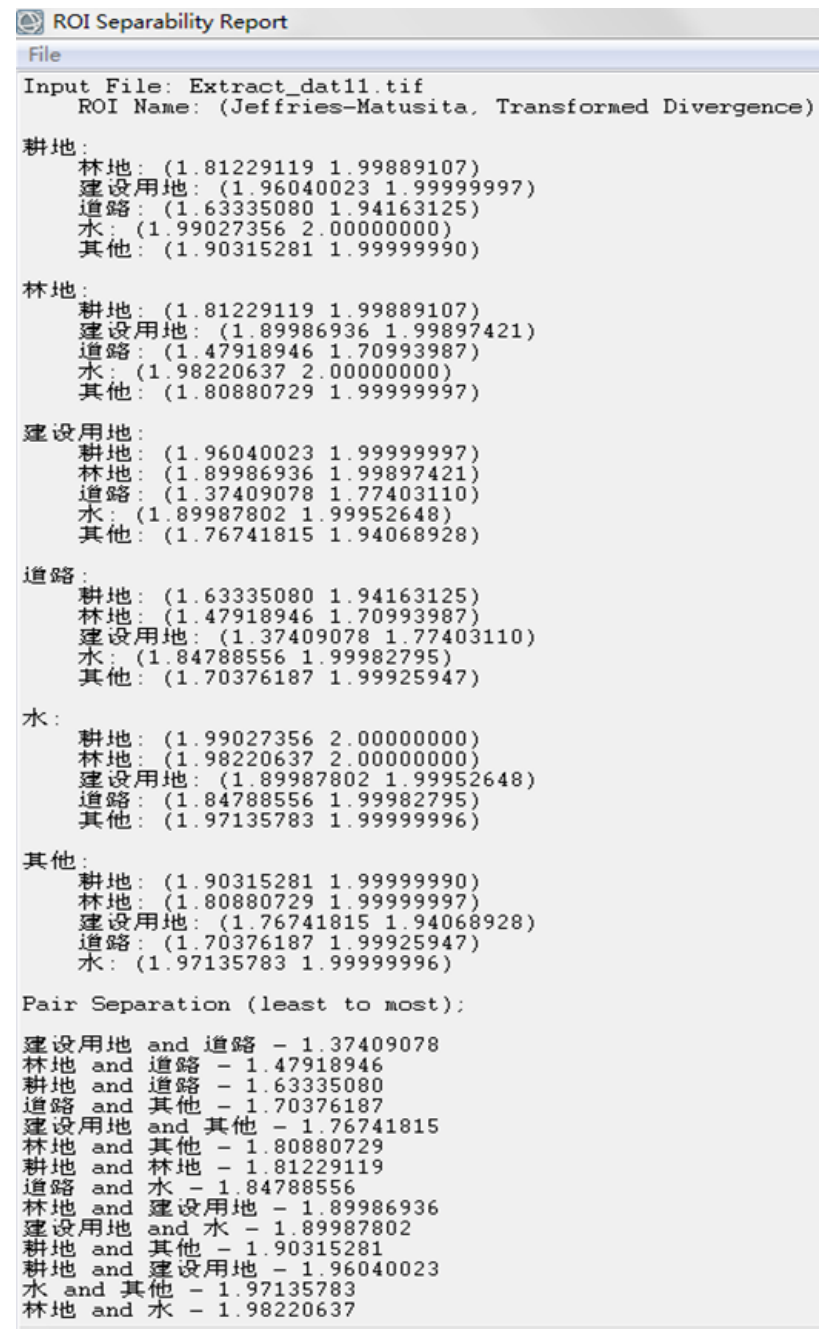

Figure 2 Evaluation of the area of interest

\subsection{Execution classification and output results}

Through the operation of ENVI software, the maximum likelihood classification and the minimum distance method for supervised classification of images, the ISODATA classification and K-means classification for unsupervised classification. In order to study the differences between different classification algorithms, the same training samples were used for supervised classification experiments. Unsupervised uses the same number of iterations and corresponding parameter values, and the classification results are shown in the figure2-5 below.

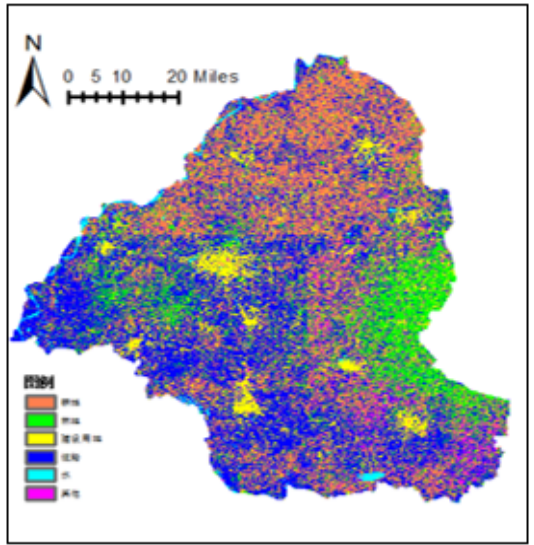

Figure 3 . Result of maximum likelihood classification results

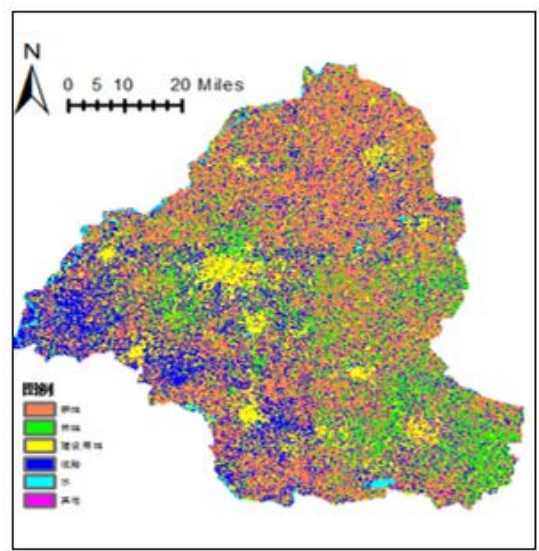

Figure 4 . Result of minimum distance classification

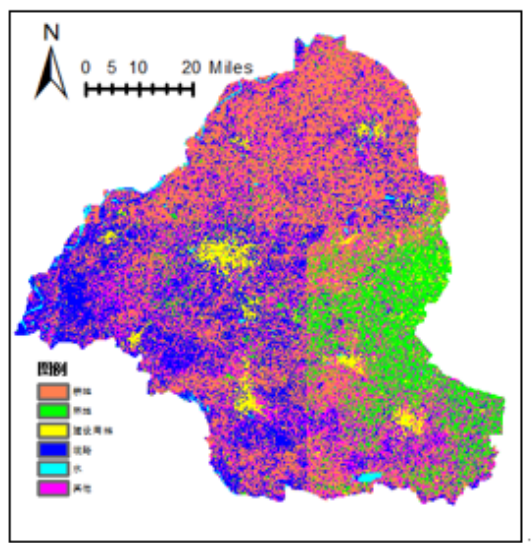

Figure 5 . Result of ISODATA classification 


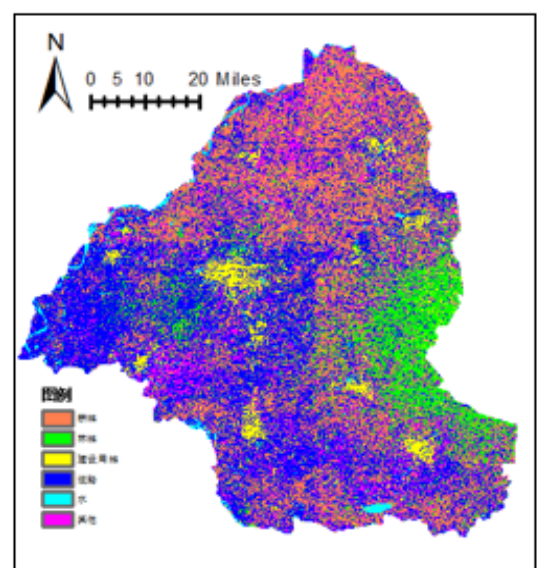

Figure 6 . Result of K-means classification

It can be seen from the above four classification results that the unsupervised classification effect is significantly worse than the supervised classification. The minimum distance method in supervised classification is worse than the maximum likelihood method. The minimum distance method is prone to misclassification of roads and construction land and cultivated land. The overall classification accuracy is $81.5952 \%$, The classification accuracy of the maximum likelihood method is 82.3281\%, which is slightly better than the minimum distance method. The error of the missing points is relatively small, see Table 2.. The classification effect of ISODATA method in unsupervised classification is better than K-means algorithm. The classification effect of K-means algorithm on cultivated land and woodland is relatively poor. It needs to pre-select the number of categories, and the number of categories directly affects the classification effect. It can be seen from the output that the K-mean leakage phenomenon is obvious and the classification effect is very poor. The ISODATA classification method is relatively good. The construction land and roads can be seen as distinct separation phenomena, and the differences in ground objects are distinguished. as shown in Table 3.

\begin{tabular}{|l|c|c|}
\hline Classification & Overall Accuracy & $\begin{array}{l}\text { Kappa } \\
\text { Coefficient }\end{array}$ \\
\hline $\begin{array}{l}\text { Maximum } \\
\text { likelihood } \\
\text { method }\end{array}$ & $82.3281 \%$ & 0.7591 \\
Minimum & $81.5952 \%$ & 0.7431 \\
\hline
\end{tabular}

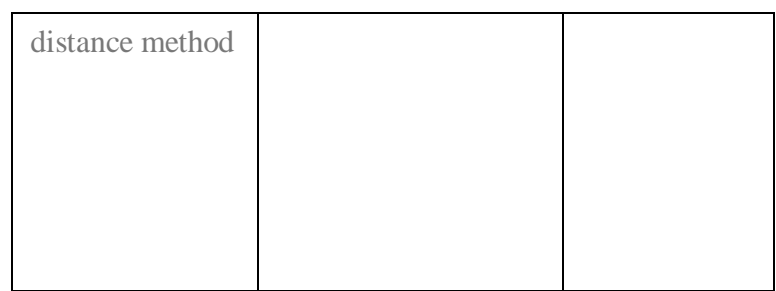

Table 2. Supervised classification accuracy

\begin{tabular}{|l|l|l|}
\hline Classification & Clustering effect & Missing points \\
\hline $\begin{array}{l}\text { ISODATA } \\
\text { method } \\
\begin{array}{l}\text { K-means } \\
\text { method }\end{array}\end{array}$ & Good & Rarely \\
\hline
\end{tabular}

Table 3. Unsupervised classification clustering effect

\section{CONCLUSIONS AND DISCUSSION}

The improvement of classification accuracy of remote sensing images has always been a hot issue. In this paper, the Landsat-8 OLI Heze region remote sensing data is based on two common supervised classifications and two unsupervised classification methods for comparative analysis and result accuracy evaluation. The main conclusions are as follows:

1) In the supervised classification method, the minimum distance method is prone to misclassification of roads, construction land and cultivated land, and the classification effect is not as good as the maximum likelihood method, but the classification effect is very good in water bodies, construction land and forest land categories. The accuracy of the maximum likelihood method classification is that the classification accuracy is relatively high and the leakage points are less. And with the wide selection of samples, the accuracy of the classification results will be higher.

2) In the unsupervised classification method, the difference between the ISODATA algorithm and the K-means algorithm is significant. There are many misclassification and leakage phenomena in the K-means algorithm. There is no obvious sense of separation of objects in the whole classification result. It is necessary to consider increasing the number of iterations or changing the parameters to improve. The ISODATA algorithm is relatively good, and the classification of construction land and water body is obvious. It is possible to visually interpret the 
differences in features and determine the type of features.

Most of the current methods have limitations in classification.

No method is the best classification. The classification method must be selected according to the spectral characteristics of the remote sensing image and the actual working requirements. Choose the right method to improve the classification accuracy.

\section{ACKNOWLEDGEMENTS}

This work was sponsored by the National Natural Foundation of China (41664002;41704027); Guangxi Natural Science Foundation of China (2018GXNSFAA294045; 2017GXNSFDA198016; 2017GXNSFBA198139); the "Ba Gui Scholars” program of the provincial government of Guangxi; and the Guangxi Key Laboratory of Spatial Information and Geomatics (14-045-24-10;16-380-25-01)

\section{REFERENCES}

Yang,C.J.,Zhou,C.H.,2010:.Discussion on Classification Method of Remote Sensing Image Based on Knowledge. Geography and Territorial Research, 17(1): 72-77

Zhang,M., Huang,S.Y., 2019:Classification of Remote Sensing Image Based on Landsat-8, Geographic and Spatial Geographic Information, 42(1): 177-180

Tong,W., 2019:Research on supervised classification method of remote sensing image based on ENVI,

Technological Innovation and Application, (23): 6-9

Wang,D.li.,Zhang,A.B.,Zhao,A.Z., et al.,2019:Unsupervised classification of winter wheat planting information extraction model, Bulletin of Surveying and Mapping, (8): 68-71

Zhang,Y.,2016:Discussion on supervised classification and unsupervised classification methods of remote sensing images, Heilongjiang Science and Technology Information, (2): 79-80

JIN,J.,ZHU,H.Y.,LI,Zi.kai., et al.,2014: Comparison of several supervised classification methods in ENVI remote sensing image processing, Water Science and Technology and Economy, 20 (01): 146-148+160.

Zhao,C.X.,Qian,L.X.,2004:Comparison of supervised classification and unsupervised classification of remote sensing images , Journal of Henan University (Natural Science Edition).

Zhao,L.,Zhou,G.H., 2016:Strawberry image segmentation based on ISODATA algorithm,Hubei Agricultural Sciences, 55 (18), $4812-4813+4852$

Lin,B.,Chen,J.,QuD.Y., et al.,2019: Research on traffic state discrimination method based on K-means clustering algorithm, Journal of Qingdao Technological University ,40(04), 109-114 